\section{(1)}

CrossMark

\title{
State of the art in interstitial pneumonia with autoimmune features: a systematic review on retrospective studies and suggestions for further advances
}

\author{
Gianluca Sambataro ${ }^{1,2}$, Domenico Sambataro ${ }^{2}$, Sebastiano Emanuele Torrisi ${ }^{1}$, \\ Ada Vancheri ${ }^{1}$, Mauro Pavone ${ }^{1}$, Roberta Rosso ${ }^{1}$, Matteo Schisano ${ }^{1}$, \\ Claudia Crimi ${ }^{1}$, Francesca Pignataro ${ }^{3}$, Aryeh Fischer ${ }^{4}$, Nicoletta Del Papa ${ }^{3}$ and \\ Carlo Vancheri ${ }^{1}$
}

Affiliations: ${ }^{1}$ Regional Referral Centre for Rare Lung Diseases, A.O.U. “Policlinico-Vittorio Emanuele" Dept of Clinical and Experimental Medicine, University of Catania, Catania, Italy. ${ }^{2}$ Rheumatology Outpatients, accredited with the National Health System, Artroreuma srl, Mascalucia, CT, Italy. ${ }^{3}$ Day Hospital of Rheumatology, Dept of Rheumatology, ASST G. Pini-CTO, Milan, Italy. ${ }^{4}$ Dept of Medicine, University of Colorado School of Medicine, Denver, CO, USA.

Correspondence: Gianluca Sambataro, Rheumatology, Artroreuma S.R.L. corso S. Vito 53, Mascalucia (CT), 95030, Italy. E-mail dottorsambatarolagmail.com

@ERSpublications

IPAF retrospective cohorts show numerous differences between them. We propose some ideas to improve IPAF criteria http://ow.ly/eubC30jlGJO

Cite this article as: Sambataro G, Sambataro D, Torrisi SE, et al. State of the art in interstitial pneumonia with autoimmune features: a systematic review on retrospective studies and suggestions for further advances. Eur Respir Rev 2018; 27: 170139 [https://doi.org/10.1183/16000617.0139-2017].

ABSTRACT The term interstitial pneumonia with autoimmune features (IPAF) has been proposed to define patients with interstitial lung disease (ILD) associated with autoimmune signs not classifiable for connective tissue diseases (CTDs). This new definition overcomes previous nomenclatures and provides a uniform structure for prospective studies through specific classification criteria.

This work evaluates the characteristics of IPAF patients reported in the literature, to highlight potential limits through a comparative analysis and to suggest better performing classification criteria.

Four retrospective studies on the IPAF population have been considered. The study subjects differed in age, sex, smoking habit, ILD pattern and outcomes. Another important difference lies in the diverse items considered in the classification criteria. The retrospective design of the studies and the absence from some of them of a rheumatologist clearly involved in the diagnosis may have influenced the data, but current IPAF criteria seem to include a rather heterogeneous population. To overcome these discrepancies, this review suggests a limitation in the use of single items and the exclusion of extremely specific CTD criteria. This should avoid the definition of IPAF for those diseases at different stages or at early onset. The investigation of a functional or morphological cut-off of pulmonary involvement would be useful.

\section{Introduction}

The interstitial pneumonias comprise a large group of diseases mainly characterised by excessive deposition of collagen matrices in the pulmonary interstitium, inducing a restrictive pattern in pulmonary function tests (PFTs). Some interstitial pneumonias are considered idiopathic, others recognise diverse risk

Received: Dec 222017 | Accepted after revision: March 292018

Provenance: Submitted article, peer reviewed.

Copyright OERS 2018. ERR articles are open access and distributed under the terms of the Creative Commons Attribution Non-Commercial Licence 4.0. 
factors, such as smoking, environmental and occupational exposures, while some others are related to connective tissue disease (CTD) [1]. An increasing number of studies have focused on the presence of a particular subset of interstitial pneumonia, apparently idiopathic, associated to one or more clinical and serological features, suggesting a possible underlying autoimmune disorder. Several definitions and nomenclatures have been proposed for this specific form of interstitial pneumonia [2-5], until the recent joint effort of the European Respiratory Society (ERS) and American Thoracic Society (ATS) proposed "interstitial pneumonia with autoimmune features" (IPAF) for this condition [6]. This broadly accepted

\section{TABLE 1 Classification criteria of interstitial pneumonia with autoimmune features}

1. Presence of an interstitial pneumonia (by HRCT or surgical lung biopsy), and

2. Exclusion of alternative aetiologies, and

3. Does not meet criteria of a defined connective tissue disease, and

4. At least one feature from at least two of these domains:
A. clinical domain
B. serological domain
C. morphological domain

A. Clinical domain

1. Distal digital fissuring (i.e. "mechanic's hands")

2. Distal digital tip ulceration

3. Inflammatory arthritis or polyarticular morning stiffness $\geqslant 60 \mathrm{~min}$

4. Palmar telangiectasia

5. Raynaud's phenomenon

6. Unexplained digital oedema

7. Unexplained fixed rash on the digital extensor surfaces (Gottron's sign)

B. Serological domain

1. $A N A \geqslant 1: 320$ titre, diffuse, speckled, homogeneous patterns or any titre for nuclear and centromere pattern

2. Rheumatoid factor $\geqslant 2 \times$ upper limit of normal

3. Anti-CCP

4. Anti-dsDNA

5. Anti-Ro (SS-A)

6. Anti-La (SS-B)

7. Anti-ribonucleoprotein

8. Anti-Smith

9. Anti-topoisomerase (Scl-70)

10. Anti-tRNA synthetase (e.g. Jo-1, PL-7, Pl-12, EJ, OJ, KS, Zo, tRS)

11. Anti-PM/Scl

12. Anti-MDA-5

C. Morphological domain

1. Suggestive radiology patterns by HRCT:

a. NSIP

b. OP

c. NSIP with OP overlap

d. LIP

2. Histopathology patterns or features by surgical lung biopsy:

a. NSIP

b. OP

c. NSIP with OP overlap

d. LIP

e. Interstitial lymphoid aggregates with germinal centres

f. Diffuse lymphoplasmacytic infiltration (with or without lymphoid follicles)

3. Multicompartment involvement (in addition to interstitial pneumonia):

a. Unexplained pleural effusion or thickening

b. Unexplained pericardial effusion or thickening

c. Unexplained intrinsic airways disease ${ }^{\#}$ (by PFT, imaging or pathology)

d. Unexplained pulmonary vasculopathy

HRCT: high-resolution computed tomography; ANA: antinuclear antibody; ds: double-stranded; SS: Sjögren syndrome; NSIP: nonspecific interstitial pneumonia; OP: organising pneumonia; LIP: lymphoid interstitial pneumonia; PFT: pulmonary function testing. " : includes airflow obstruction, bronchiolitis or bronchiectasis. Reproduced from [6] with permission. 
definition was needed to allow prospective data collection for further studies (complete IPAF classification criteria are reported in table 1).

Unfortunately, to date, none of the proposed definitions, focusing on interstitial pneumonia associated with a variety of autoimmune features, consider that pulmonary involvement may be the prevalent, the primary or even the only clinical manifestation of CTDs [7]. In this subset of patients, there may be interstitial pneumonias with some clinical and/or serological characteristics of autoimmune disorders even if they do not satisfy any specific CTD criteria. Indeed, there is a fine line between interstitial pneumonias with autoimmune features and undifferentiated connective tissue diseases (UCTDs).

UCTDs include unclassifiable systemic autoimmune diseases that share clinical and serological features with CTDs, such as systemic lupus erythematosus, systemic sclerosis, Sjögren syndrome, dermatomyositis/ polymyositis, mixed connective tissue diseases, anti-synthetase syndrome (ASS) and rheumatoid arthritis. Nevertheless, UCTDs do not correspond to any of the specific existing classification criteria [8-15]. The current definitions of UCTD are not yet validated and UCTD is still waiting for well-established classification criteria [16, 17]. Thus, differentiating IPAF from UCTDs would be crucial to better understand the natural history of the diseases, to identify a correct therapeutic approach and to lay the foundation for additional research.

The primary aim of this work was to review the retrospective cohorts of patients classified according to IPAF criteria presented in the literature and evaluate their specific characteristics. Secondarily, we discuss their role in the setting of clinical research and their function as classification criteria.

\section{Materials and methods \\ Literature search strategy}

Two authors independently performed a systematic search of PubMed, Web of Science, the Cochrane database, EBSCO and BioMed Central. Comprehensive searching used the medical subject heading $(\mathrm{MeSH})$ terms "interstitial pneumonia with autoimmune features" and "IPAF" to identify all descriptive works on IPAF patients subsequent to publishing specific criteria, between January 2015 to October 2017. The bibliography of every cited study was checked to identify any additional study suitable for review. We selected only original research articles describing $\geqslant 70 \%$ of IPAF items. Case reports, case series, letters to editors and reviews were not analysed. From three different articles dealing with the same cohort of IPAF patients, only the one with complete data was considered [18-20]. Finally, manuscripts considering ASS patients were excluded to avoid any selection bias. In spite of the absence of validated classification criteria, ASS is a specific CTD marked by the classic triad composed of ILD, inflammatory arthritis and myositis. Although the clinical triad may be partially present at the time of diagnosis, the majority of patients subsequently develop all clinical signs of ASS during follow-up [21].

\section{Data extraction and quality assessment}

Two authors (GS and DS) independently browsed titles, abstracts and full texts meeting the defined criteria. Any discrepancy was resolved by consensus with senior authors (NDP and CV). Each study's clinical characteristics were summarised, and each study's main outcomes were extracted or calculated. The process is reported in figure 1 .

\section{Statistical analysis}

All statistical analyses were performed using Prism 7.0 software (GraphPad, San Diego, CA, USA). Continuous variables were compared using t-tests. $z$-tests were used to compare population proportions to identify any difference in clinical characteristics or in items of IPAF classification criteria.

\section{Results}

Four studies satisfied the selection criteria, reporting complete clinical, serological and morphological characteristics of IPAF patients.

In the first study, OLDHAm et al. [18] identified 144 patients affected by IPAF from a population of 422 suffering from idiopathic interstitial pneumonia or CDT-ILD. This IPAF population had a mean age of 63 years; about half of them presented usual interstitial pneumonia (UIP) as prevalent radiological and/or histological pattern of idiopathic interstitial pneumonia. Complete clinical features are reported in table 2. For classification criteria, $14.6 \%$ of the population met both clinical and serological domains, $8.3 \%$ presented clinical and morphological domains and $26.4 \%$ met all three domains. An overall analysis showed a mortality for IPAF similar to idiopathic pulmonary fibrosis (IPF) (39.6\% died and $10.8 \%$ underwent lung transplantation). However, stratifying for prevalent high-resolution chest tomography (HRCT) pattern, only UIP-IPAF resembled IPF, while non-UIP-IPAF showed a significantly better 
FIGURE 1 Inclusion and exclusion of publications identified through database searches. IPAF: interstitial pneumonia with autoimmune features.

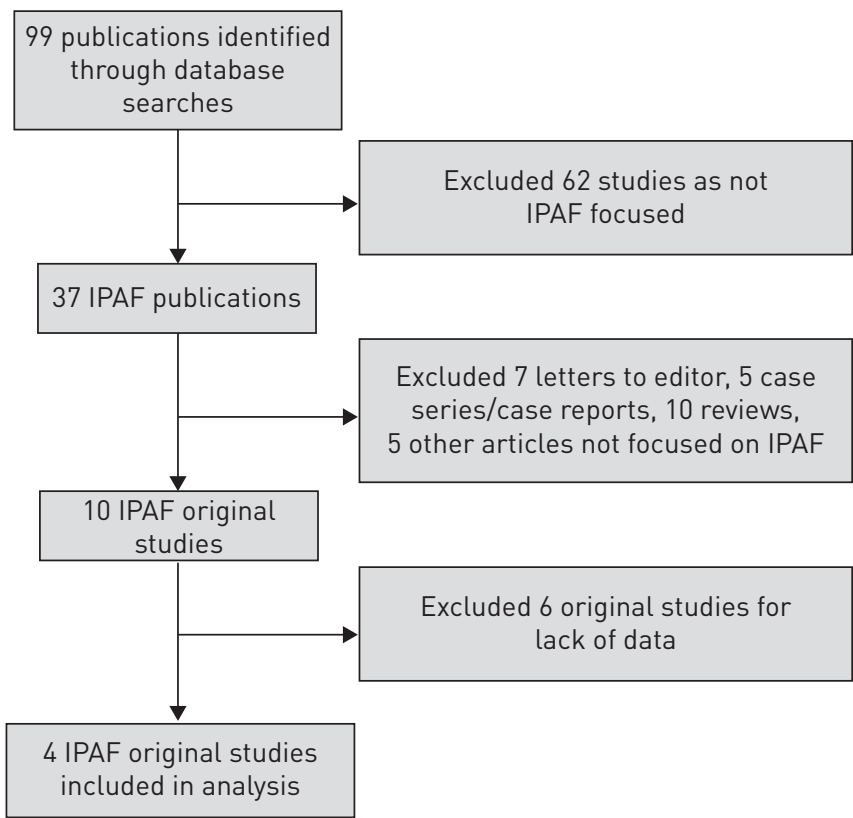

outcome, similar to CTD-ILD. In the multivariate analysis, age and diffusing capacity of the lung for carbon monoxide were associated with worse prognosis.

CHARTRAND et al. [22] reported an IPAF population of 56 patients, with a mean age of 54.6 years and mainly nonsmokers. The prevalent HRCT and surgical lung biopsy pattern resulted in nonspecific interstitial pneumonia (NSIP) (51.8\%), and UIP was reported in $\sim 9 \%$ of patients. More than half of patients met criteria for all three domains, $37.5 \%$ of patients were classified for serological and morphological domains, 9\% were classified for clinical and morphological domains and $1.5 \%$ (only one patient) classified for serological and clinical domains. The most frequent clinical features were Raynaud's phenomenon (39.3\%), mechanic's hands (28.6\%) and Gottron's sign (17.9\%). In the serological domain, the most common positive features were antinuclear antibody (ANA) (48.2\%), anti-Ro/Sjögren syndrome-A (42.9\%) and the anti-tRNA synthetase antibody (ATSA) family (35.7\%). As for outcomes, the authors reported no mortality during follow-up (duration of $284.9 \pm 141.3$ weeks) without any new clinical or serological criteria for specific CTDs.

Ahmad et al. [23] described a population of 57 IPAF patients. Among them, 11.1\% showed clinical and serological signs, $7 \%$ clinical and morphological signs, 52.7\% serological and morphological signs and $29.2 \%$ showed signs from all three domains. IPAF patients presented a mean age of $64.4 \pm 14$ years with no sex predominance, and $34 \%$ of them were smokers. The most frequent HRCT patterns were NSIP (42.1\%) and UIP (28\%), whereas the most frequent multicompartment involvement was pulmonary vasculopathy (17.5\%). Over a median follow-up period of 16 months, seven patients died. The multivariate analysis revealed only smoking habit as a predictive factor of worse prognosis and no significant difference between UIP and NSIP pattern.

The last contribution, produced by ITO et al. [24], investigated 99 patients with IPAF. The only patient with a UIP pattern and at least one clinical and serological criterion for IPAF was excluded. Among the residual 98 patients, $64.3 \%$ showed an NSIP pattern, $20.4 \%$ organising pneumonia and the remaining $15.3 \%$ an NSIP+OP pattern. This work was mainly directed at understanding the prognosis of these patients, describing a 5 -year survival of $71.1 \%$ with a median of 12.5 years. The negative prognostic factors were age and NSIP pattern, rather than the presence of specific antibodies. Moreover, 12 (12.2\%) patients developed a definite CTD (mainly rheumatoid arthritis).

\section{Comparative analysis}

Our analysis included 355 patients affected by IPAF (257 matching the clinical domain). Comparative analyses of the studies showed significant differences between the IPAF cohorts.

Chartrand et al. [22] described the youngest population $(\mathrm{p}<0.0001$ compared with others), and the highest percentage of females ( $\mathrm{p}=0.03$ versus OlDHAm et al. [18] and $\mathrm{p}=0.01$ versus Ahmad et al. [23], but similar to Iто et al. [24]). In the study by Oldham et al. [18], the population included the highest proportion of smokers ( $\mathrm{p}=0.03$ versus Chartrand et al. [22], $\mathrm{p}=0.01$ versus Ahmad et al. [23] and $\mathrm{p}=0.01$ versus Iто et al. [24]) and the lowest proportion of patients treated with corticosteroids $(\mathrm{p}<0.0001)$. 
TABLE 2 Patient clinical characteristics and clinical domains reported in studies

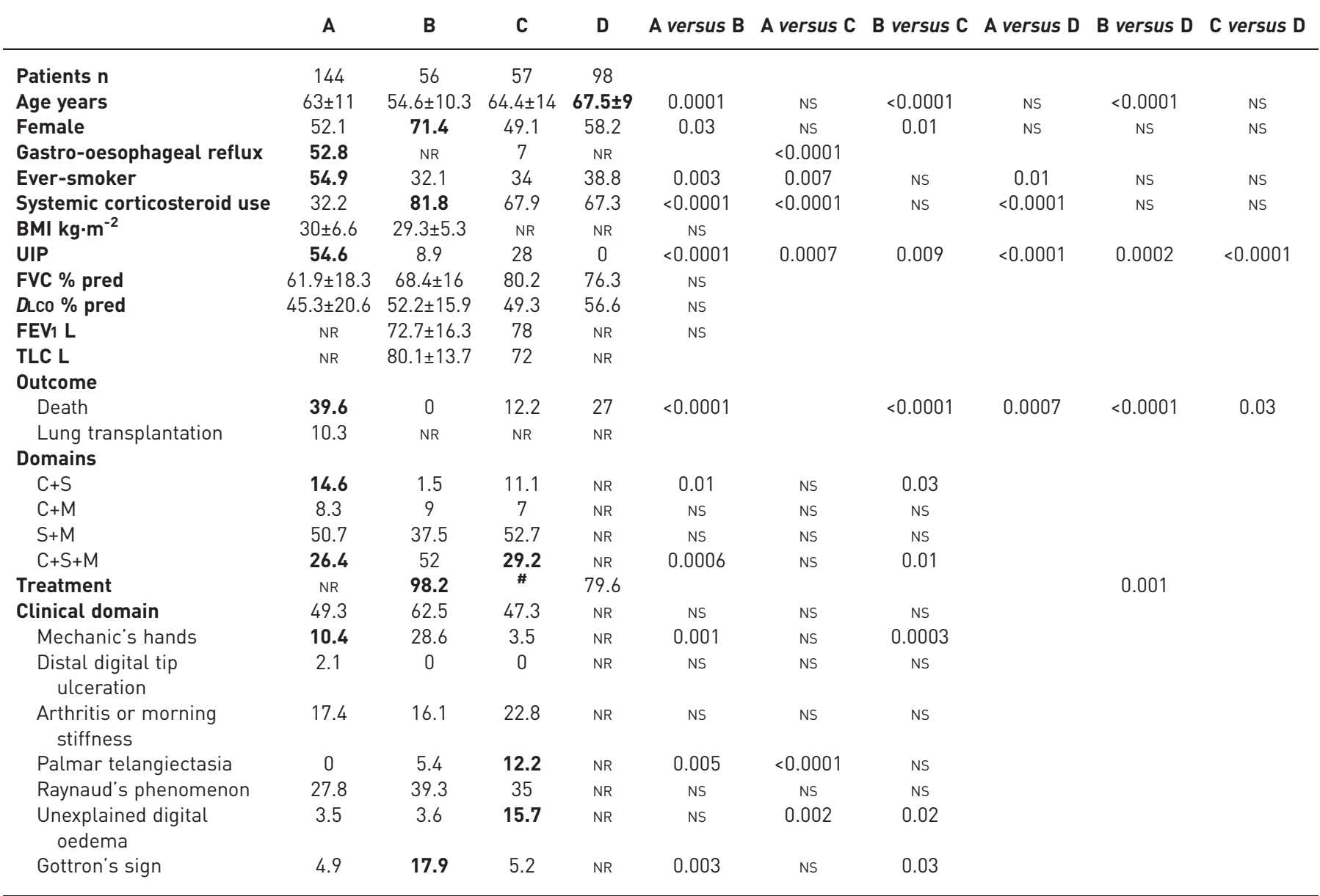

Data are presented as mean \pm SD or $\%$, unless otherwise stated. Bold represents the highest significant value. A: OLDHAM et al. [18]; B: ChARTRAND et al. [22]; C: AHMAD et al. [23]; D: ITo et al. [24]; BMI: body mass index; UIP: usual interstitial pneumonia; FVC: forced vital capacity; DLCo: diffusing capacity of the lung for carbon monoxide; FEV1: forced expiratory volume in $1 \mathrm{~s}$; TLC: total lung capacity; C: clinical; S: serological; M: morphological; NS: nonsignificant; NR: not reported. " : not clearly reported.

Regarding UIP pattern, each study is different from the others, with the highest percentage in the population of Oldham et al. [18] population and the lower in the study by Iто et al. [24] (table 2).

The populations in the studies by OLDHAm et al. [18] and AHMAD et al. [23] showed significantly higher proportions of patients meeting the clinical and serological criteria ( $\mathrm{p}=0.01$ and $\mathrm{p}=0.03$, respectively) and significantly lower proportions of patients presenting all three domains $(p=0.0006$ and $p=0.01$, respectively) compared to CHARTRAND et al. [22] (table 2).

Taking into account the IPAF criteria in clinical domains, the population in the study by CHARTRAND et al. [22] showed a higher proportion of mechanic's hand and Gottron's sign compared to those by AHMAD et al. [23] and Oldham et al. [18] $(\mathrm{p}=0.0003$ and $\mathrm{p}=0.001$; and $\mathrm{p}=0.03$ and $\mathrm{p}=0.003$, respectively). A complete comparison is reported in table 2 .

Considering the serological domain, the population in the study by CHARTRAND et al. [22] showed the lowest proportion of ANA positivity (considering a minimum titre of 1:320 for any pattern and any titre for centromeric and nucleolar patterns) $(\mathrm{p}<0.0001$ compared to Oldham et al. [18] and ITO et al. [24]; $\mathrm{p}=0.0001$ compared to Ahmad et al. [23]). Conversely, the population in CharTRAnd et al. [22] showed a significantly higher proportion of anti-Ro/Sjögren syndrome-A, anti-RNP, anti-Smith and ATSA antibodies compared to the other studies (table 3). Unlike other investigators, Iто et al. considered rheumatoid factor to be positive when it was three times (not twice, as reported in IPAF criteria) the cut-off value; even so, they presented the highest proportion of rheumatoid factor.

For the morphological domain, the populations in the studies by Chartrand et al. [22] and Ito et al. [24] presented higher proportions of patients with significantly higher presence of NSIP patterns $(p=0.009$ 
TABLE 3 Comparison of serological domains

\begin{tabular}{|c|c|c|c|c|c|c|c|c|c|c|}
\hline & A & B & C & D & A versus $B$ & A versus C & B versus C & A versus D & B versus D & C versus D \\
\hline Serological domain & 91.7 & 91.1 & 93 & $N R$ & NS & NS & NS & & & \\
\hline $\mathrm{RF} \geqslant 40 \mathrm{IU}$ & 13 & 10.7 & 7 & $28.5^{\#}$ & NS & NS & NS & 0.0002 & 0.01 & 0.0001 \\
\hline ACPA & 4.7 & 10.7 & 8.8 & 15.3 & NS & NS & NS & 0.004 & NS & NS \\
\hline Anti-Sdna & 7.2 & 1.8 & 5.2 & 6.2 & NS & NS & NS & NS & NS & NS \\
\hline Anti-Ro/SS-A & 16.6 & 42.9 & 8.8 & 18.3 & $<0.0001$ & NS & $<0.0001$ & NS & 0.001 & NS \\
\hline Anti-RNP & 4.9 & 16.1 & 0 & 9.1 & 0.008 & NS & 0.001 & NS & NS & 0.01 \\
\hline Anti-Smith & 1.5 & 8.9 & 0 & 4.1 & 0.01 & NS & 0.02 & NS & NS & NS \\
\hline Anti-Scl70 & 3 & 1.8 & 5.2 & 8.1 & NS & NS & NS & 0.07 & NS & NS \\
\hline Anti-tRNA synthetase & 0.7 & 35.7 & 15.8 & 13.2 & $<0.0001$ & 0.05 & 0.01 & $<0.0001$ & 0.001 & NS \\
\hline Anti-PM/Scl & & 1.8 & 5.2 & NR & & & NS & & & \\
\hline Anti-MDA-5 & & 0 & 0 & NR & & & NS & & & \\
\hline
\end{tabular}

Data are presented as mean \pm SD or $\%$, unless otherwise stated. Bold represents the highest significant value. A: OLDHAM et al. [18]; B: ChaRTRAND et al. [22]; C: AHMAD et al. [23]; D: Ito et al. [24]; ANA: antinuclear antibodies; RF: rheumatoid factor; ACPA: anti-citrullinated protein antibodies; SS: Sjögren syndrome; RNP: ribonucleoprotein; PM/Scl: polymyositis/systemic sclerosis; MDA: melanoma differentiation antigen; NR: not reported; NS: nonsignificant. ${ }^{\#}$ : RF >60 IU.

compared to Oldham et al. [18] and p=0.001 compared to AHMAD et al. [23]) (complete comparison is presented in table 4). Finally, for patient outcomes, as expected considering the higher proportion of UIP patients, the population in the study by OLDHam et al. [18] showed the highest mortality rate (39.6\%) compared to those in the studies by ChARTRAND et al. [22] $(\mathrm{p}<0.0001)$ and ITO et al. [24] $(\mathrm{p}=0.0007)$, but the latter population, despite a comparable HRCT pattern, showed significantly worse prognosis compared to the population in the study by Chartrand et al. [22] $(\mathrm{p}<0.0001$, table 2$)$. In addition, differences in terms of treatment were reported. The population in the study by CHARTRAND et al. [22] shows the highest percentage of treated patients $(\mathrm{p}=0.001)$.

In addition, Oldham et al. [18] performed a further stratification by HRCT pattern. Non-UIP IPAF patients had a prognosis very similar to those with CTD-ILD, whereas the progression of the disease in UIP-IPAF patients was resembled IPF.

However, these data were not confirmed by AHMAD et al., who reported a similar prognosis for IPAF and IPF patients.

TABLE 4 Comparison of morphological domains

\begin{tabular}{|c|c|c|c|c|c|c|c|c|c|c|}
\hline & A & B & C & D & A versus $\mathrm{B}$ & A versus $\mathrm{C}$ & B versus C & A versus D & B versus D & C versus D \\
\hline Patients n & 144 & 56 & 57 & 98 & & & & & & \\
\hline Morphological domain & 85.4 & 98.2 & 78.9 & 100 & 0.009 & NS & 0.001 & 0.0001 & NS & $<0.0001$ \\
\hline $\mathrm{OP}$ & 3.5 & 3.6 & 3.5 & 20.4 & NS & NS & NS & $<0.0001$ & 0.004 & 0.003 \\
\hline $\mathrm{NSIP}+\mathrm{OP}$ & 7.8 & 17.9 & 15.8 & 15.3 & 0.001 & NS & NS & NS & NS & NS \\
\hline UIP & $\mathrm{NR}$ & 5.4 & 1.8 & 0 & & & NS & & 0.02 & NS \\
\hline Pericardial effusion or thickening & 1.4 & 1.8 & 1.8 & NR & NS & NS & NS & & & \\
\hline Intrinsic airways disease & 22.2 & 12.5 & 8.8 & NR & NS & 0.02 & NS & & & \\
\hline Pulmonary vasculopathy & 18.8 & 30.4 & 17.5 & NR & NS & NS & NS & & & \\
\hline
\end{tabular}

Data are presented as mean \pm SD or $\%$, unless otherwise stated. Bold represents the highest significant value. A: OLDHAM et al. [18]; B: ChARTRAND et al. [22]; C: AHMAD et al. [23]; D: Ito et al. [24]; NSIP: nonspecific interstitial pneumonia; OP: organising pneumonia; UIP: usual interstitial pneumonia; HRCT: high-resolution chest tomography; SLB: surgical lung biopsy; NS: nonsignificant; NR: not reported. 


\section{Discussion}

Classification criteria are standardised definitions with the primary aim of creating homogeneous cohorts for research [25]. In this way, they favour high specificity over sensitivity. In 2015, the IPAF criteria [6] were created via consensus opinion by an expert international panel of 13 pulmonologists, four rheumatologists, one thoracic radiologist and one pulmonary pathologist. Importantly, the panel was largely composed by the same investigators who had previously put forth criteria for ILD with autoimmune characteristics. The IPAF criteria, endorsed by the ATS and ERS proposed a research classification for interstitial pneumonia patients who presented at least one specific feature from at least two of the following three domains: clinical, serological and morphological. The proposed IPAF classification has the advantage of removing previous nomenclatures, creating a framework to study a more uniform population in a readily applicable manner worldwide.

Comparing the already reported IPAF cohorts, in our analysis we identified very different populations from the clinical, serological and morphological point of view. In addition, some differences were noted in terms of outcomes. As expected, mortality was higher in the OLDHAM et al. [18] cohorts, where more patients had a UIP pattern. However, in the other studies, mortality was not strictly related to the presence of a UIP pattern.

Obviously, comparison of different studies may have limitations. The first one is related to the retrospective nature of the reviewed cohorts, which can, at least in part, explain the numerous differences highlighted observed among them. In fact, IPAF criteria were proposed for prospective studies and data from these will be of pivotal interest. A second limitation is related to the lack of a rheumatologist clearly involved in the multidisciplinary team (MDT). CHARTRAND et al. [22] reported the presence of an expert rheumatologist in the diagnostic process, whereas in the other studies this role was not clearly involved in diagnosis. This difference may explain the reason why the cohort in the CHARTRAND et al. study has features and outcomes similar to CTD, whereas patients in the OLDHAM et al. cohort have features and outcomes more similar to IPF. A third weakness concerns treatment. OLDHAM et al. did not report any patient treatment. The other studies gave only a partial description of how patients were treated. In some cases, they were treated with steroids and/or immunosuppressant drugs according to the low prevalence of UIP pattern. Furthermore, despite the similarity of the HRCT pattern in the ITo et al. [24] and CharTrand et al. populations, a low percentage of treated patients and a worse prognosis was observed in the former cohort. These data may suggest the importance of treatment in the evolution and prognosis of IPAF patients.

Nevertheless, in the absence of proper prospective studies, the comparison and analysis of these four retrospective studies give some useful insights that may be helpful to improve the current IPAF classification.

As described, IPAF criteria overlap UCTD criteria as well as those criteria that identify early onset or incomplete forms of defined CTDs $[16,17,26]$. These disorders might be called "IPAF" inappropriately, while they represent different diseases at different stages. For instance, criteria for very early diagnosis of systemic sclerosis (VEDOSS) [26] are able to identify individuals with a high risk of developing a defined systemic sclerosis. These patients are of great interest, considering that their evident "sclerodermic flavour" allows the timing of onset of the disease to be studied, or whether the presence or even the absence of an item in the pathogenic behaviour influences complete disease manifestation.

Although criteria for very early diagnosis of other CTDs are not yet defined, IPAF criteria can capture all these forms under a unique definition. In this context, it should be considered that different CTDs, even at early stages, have different pathogenic pathways and varying evolution.

Moreover, IPAF criteria may include patients with ASS. Patients with a positive ATSA and ILD could be considered to have ASS by some clinicians and IPAF by others, despite the high risk of developing other clinical findings of the classic triad (arthritis and/or myositis) [21]. In this regard, it seems important to consider the mutual concept: ILD patients should be tested for all ATSA and other antibodies linked to inflammatory myopathies, although some of these antibodies are not widely available.

Thus, the current definition of IPAF is not probably able to select homogenous populations. On the contrary, in the context of the MDT follow-up, IPAF classification is useful in selecting subsets of ILD patients at risk of developing a defined CTD.

Another point of view could be that IPAF is a primary autoimmune disease, which mainly focuses on the lung. To sustain this hypothesis, it is necessary to satisfy Koch's postulate for autoimmune diseases [27] by demonstrating autoreactive T- or B-cells (specific antibodies). In support of this thesis, IPAF populations show higher proportions of ANA positivity than expected in the general population [28]. Moreover, antibodies useful for diagnosing CTD showed their primary autoantigen sources in the lung, such as Jo-1 and anti-citrullinated protein antibody (ACPA) [29-31]. In some other cases, antibodies are associated 
with prevalent lung involvement, such as ATSA and anti-Th/To [32, 33], and in IPF autoantibodies are less rare $[34,35]$.

In our opinion, IPAF criteria should follow the latter hypothesis to avoid the inappropriate inclusion of IPAF patients with several different clinical conditions.

In this light, the presence in these criteria of very specific items (such as Extractable Nuclear Antigen (ENA) specificities, anti-double stranded DNA, ACPA, distal digital tip ulceration, mechanic's hands) or even pathognomonic features, such as Gottron's sign [36], for definite CTDs appear to be not useful. Similarly, in spite of the lack of classification criteria for ASS, patients with ILD and myositis show positivity for ATSA up to $60 \%$ of cases [15]. Therefore, it may not be by chance that the CHARTRAND et al. [22] population showed simultaneously a higher prevalence of Gottron's sign, mechanic's hands, NSIP pattern and ATSA, four features all linked to inflammatory myopathies.

Conversely, other antibodies associated with a wide spectrum of autoimmune diseases and ILD such as anti-neutrophil cytoplasmic antibodies (ANCA) [37] and anti-Ku antibody should be considered [38, 39]. Furthermore, some reports described patients with ANCA-positive interstitial pneumonia. Currently, limited data are available for this subset of patients. YAMADA [40] reviewed the reported cohorts, describing 92 patients with interstitial pneumonia generally classifiable as IPF with positivity for ANCA. In this cohort, 35.8\% of patients showed concurrent ANCA-associated vasculitis (AAV), 17.4\% developed an AAV with a delay of years compared to the interstitial pneumonia and the remaining $46.8 \%$ showed only ANCA-positive interstitial pneumonia. These patients had a prevalence of honeycombing in surgical lung biopsy with a prognosis similar to IPF. Few other patients had signs of bronchiolar or interstitial vasculitis. The author hypothesised two pathogenic mechanisms. The first is that subclinical alveolar haemorrhage can contribute to interstitial pneumonia in AAV. The second mechanism considered the high prevalence of anti-myeloperoxidase (MPO) ANCA in these patients. In response to inflammatory cytokines, MPO is shown to the surface of neutrophils, and can be bound by circulating ANCA, with subsequent neutrophil degranulation and release of reactive oxygen species, leading to injury and fibrosis. This second mechanism might explain ANCA-associated interstitial pneumonia without vasculitis.

In addition, this optimisation of the number of diagnostic items could avoid some confusion in classification. Particular attention should be paid to the possible association of autoimmune features during the classification of a single patient. In several cases, patients with combined positivity for high titre of rheumatoid factor and ACPA were considered to have IPAF. This combination exposes general and high-risk populations to a $100 \%$ likelihood of developing rheumatoid arthritis in 5 years [41]. Furthermore, in the AHмAD et al. [23] population, patients with sicca syndrome and minor salivary gland biopsy suggestive of Sjögren syndrome were classified as having IPAF. In these cases, the likelihood of developing technically detectable clinical impairment of salivary or lacrimal glands and fulfilling Sjögren syndrome criteria is very high. Besides, in the same study, patients with Raynaud's phenomenon, giant capillaries at nailfold videocapillaroscopy (NVC) and NSIP pattern were classified as having IPAF rather than VEDOSS. The authors specified that NVC was considered positive for minor alterations [42, 43], but giant capillaries, as reported in the manuscript, is one of the most specific items for CTD in NVC, with well documented significance in diagnosis and prognosis [44-47]. As such, abnormal NVC may be considered as an exclusion criterion for IPAF classification.

An improvement in specificity can be obtained with a more precise definition of the morphological domain. For example, it could be enriched adding a quantification cut-off in terms of PFTs. This may explain the frequent variability of ILD in CTDs reported in the literature. An interesting study on rheumatoid arthritis reported ILD in $19 \%$ of patients, but only $6 \%$ of these showed a restrictive PFT pattern [48]. In the remaining patients, it cannot be excluded that the aetiological cause of ILD may not be autoimmune and already solved at diagnosis. The use of thresholds may improve specificity at the expense of the sensitivity of the IPAF criteria, with more chances to create a homogeneous population.

Moreover, the concept of "unexplained intrinsic airways disease" could benefit from clarification, especially regarding bronchiectasis, reported as an example of unexplained intrinsic airways disease. Its finding is extremely common in the ILDs, especially in the UIP pattern [49], hence this parameter is not useful to discriminate among patients.

In the light of these considerations, IPAF criteria could be potentially refined as follows. 1) Exclusion of items extremely specific for CTD and simplification of criteria; 2) inclusion in the serological domain of ANCA and anti-Ku antibodies; 3) evaluation of positive NVC as an exclusion criterion of IPAF; 4) definition of a PFT cut-off to consider ILD as clinically significant/at risk of progression; 5) clarification of the multicompartment involvement section (mainly intrinsic airway disease); and 6) active involvement of a rheumatologist in the MDT. 
Conflict of interest: C. Vancheri reports grants and personal fees from Roche and Boehringer Ingelheim, outside the submitted work. A. Fischer reports personal fees (consultancy and steering committee) from Boehringer Ingelheim and F Hoffman La Roche, during the conduct of the study.

\section{References}

1 Travis WD, Costabel U, Hansell DM, et al. An official American Thoracic Society/European Respiratory Society statement: update of the international multidisciplinary classification of idiopathic interstitial pneumonias. Am J Respir Crit Care Med 2013; 188: 733-743.

2 Kinder BW, Collard HR, Koth L, et al. Idiopathic nonspecific interstitial pneumonia: lung manifestation of undifferentiated connective tissue disease? Am J Respir Crit Care Med 2007; 176: 691-697.

3 Corte TJ, Copley SJ, Desai SR, et al. Significance of connective tissue disease features in idiopathic interstitial pneumonia. Eur Respir J 2012; 39: 661-668.

4 Fischer A, West SG, Swigris JJ, et al. Connective tissue disease-associated interstitial lung disease: a call for clarification. Chest 2010; 138: 251-256.

5 Vij R, Noth I, Strek ME. Autoimmune-featured interstitial lung disease: a distinct clinical entity. Chest 2011; 140: 1292-1299.

6 Fischer A, Antoniou KM, Brown KK, et al. An official European Respiratory Society/American Thoracic Society research statement: interstitial pneumonia with autoimmune features. Eur Respir J 2015; 46: 976-987.

7 Tzelepis GE, Toya SP, Moutsopoulos HM. Occult connective tissue diseases mimicking idiopathic interstitial pneumonias. Eur Respir J 2008; 31: 11-20.

8 Aletaha D, Neogi T, Silman AJ, et al. 2010 rheumatoid arthritis classification criteria: an American College of Rheumatology/European League Against Rheumatism collaborative initiative. Arthritis Rheum 2010; 62: $2569-2581$.

9 Petri M, Orbai AM, Alarcón GS, et al. Derivation and validation of the Systemic Lupus International Collaborating Clinics classification criteria for systemic lupus erythematosus. Arthritis Rheum 2012; 64: 2677-2686.

10 van den Hoogen F, Khanna D, Fransen J, et al. 2013 classification criteria for systemic sclerosis: an American College of Rheumatology/European League Against Rheumatism collaborative initiative. Arthritis Rheum 2013; 65: $2737-2747$.

11 Shiboski CH, Shiboski SC, Seror R, et al. 2016 American College of Rheumatology/European League Against Rheumatism classification criteria for primary Sjögren's syndrome: a consensus and data-driven methodology involving three international patient cohorts. Ann Rheum Dis 2017; 76: 9-16.

12 Bohan A, Peter JB. Polymyositis and dermatomyositis (first of two parts). N Engl J Med 1975; 292: 344-347.

13 Bohan A, Peter JB. Polymyositis and dermatomyositis (second of two parts). N Engl J Med 1975; 292: 403-407.

14 Alarcón-Segovia D, Cardiel MH. Comparison between 3 diagnostic criteria for mixed connective tissue disease. Study of 593 patients. J Rheumatol 1989; 16: 328-334.

15 Mahler M, Miller FW, Fritzler MJ. Idiopathic inflammatory myopathies and the anti-synthetase syndrome: a comprehensive review. Autoimmun Rev 2014; 13: 367-371.

16 Mosca M, Neri R, Bombardieri S. Undifferentiated connective tissue diseases (UCTD): a review of the literature and a proposal for preliminary classification criteria. Clin Exp Rheumatol 1999; 17: 615-620.

17 Doria A, Mosca M, Gambari PF, et al. Defining unclassifiable connective tissue diseases: incomplete, undifferentiated or both? J Rheumatol 2005; 32: 213-215.

18 Oldham JM, Adengunsoye A, Valenzi E, et al. Characterisation of patients with interstitial pneumonia with autoimmune features. Eur Respir J 2016; 47: 1767-1775.

19 Adegunsoye A, Oldham JM, Valenzi E, et al. Interstitial pneumonia with autoimmune features: value of histopathology. Arch Pathol Lab Med 2017; 141: 960-969.

20 Chung JH, Montner SM, Adengusoye A, et al. CT findings, radiologic-pathologic correlation, and imaging predictors of survival for patients with interstitial pneumonia with autoimmune features. AJR Am J Roentgenol 2017; 208: 1229-1236.

21 Scirè CA, Gonzalez-Gay MA, Selva-O'Callaghan A, et al. Clinical spectrum time course of interstitial pneumonia with autoimmune features in patients positive for antisynthetase antibodies. Respir Med 2017; 132: 265-266.

22 Chartrand S, Swigris JJ, Stanchev L, et al. Clinical features and natural history of interstitial pneumonia with autoimmune features: a single center experience. Respir Med 2016; 119: 150-154.

23 Ahmad K, Barba T, Gamondes D, et al. Interstitial pneumonia with autoimmune features: clinical, radiologic and histological characteristics and outcome in a series of 57 patients. Respir Med 2017; 123: 56-62.

24 Ito Y, Arita M, Kumagai S, et al. Serological and morphological prognostic factors in patients with interstitial pneumonia with autoimmune features. BMC Pulm Med 2017; 17: 111.

25 Aggarwal R, Ringold S, Khanna D, et al. Distinctions between diagnostic and classification criteria? Arthritis Care Res 2015; 67: 891-897.

26 Matucci-Cerinic M, Allanore Y, Czirják L, et al. The challenge of early systemic sclerosis for the EULAR Scleroderma Trial and Research Group (EUSTAR) community. It is time to cut the Gordian knot and develop a prevention or rescue strategy. Ann Rheum Dis 2009; 68: 1377-1380.

27 Pisetsky DS. Fulfilling Koch's postulates of autoimmunity: anti-NR2 antibodies in mice and men. Arthritis Rheum 2006; 8: 2349-2352.

28 Tan EM, Feltkamp TE, Smolen JS, et al. Range of antinuclear antibodies in 'healthy individuals'. Arthritis Rheum 1997; 40: 1601-1611.

29 Fischer A, Swigris JJ, du Bois RM, et al. Anti-synthetase syndrome in ANA and anti-Jo-1 negative patients presenting with idiopathic interstitial pneumonia. Respir Med 2009; 103: 1719-1724.

30 Levine SM, Raben N, Xie D, et al. Novel conformation of histidyl-transfer RNA synthetase in the lung: the target tissue in Jo1 autoantibody-associated myositis. Arthritis Rheum 2007; 56: 2729-2739.

31 Saag KG, Cerhan JR, Kolluri S, et al. Cigarette smoking and rheumatoid arthritis severity. Ann Rheum Dis 1997; 56: $463-469$.

32 Watanabe K, Handa T, Tanizawa $\mathrm{K}$, et al. Detection of antisynthetase syndrome in patients with idiopathic interstitial pneumonias. Respir Med 2011; 105: 1238-1247. 
33 Ceribelli A, Cavazzana I, Franceschini F, et al. Anti-Th/To are common antinucleolar autoantibodies in Italian patients with scleroderma. J Rheumatol 2010; 37: 2071-2075.

34 Kahloon RA, Xue J, Bhargava A, et al. Patient with idiopathic pulmonary fibrosis with antibodies to heat shock protein 70 have poor prognoses. Am J Respir Crit Care Med 2013; 187: 768-775.

35 Meliconi R, Bestagno M, Sturani C, et al. Autoantibodies to DNA topoisomerase II in cryptogenic fibrosing alveolitis and connective tissue disease. Clin Exp Immunol 1989; 76: 184-189.

36 Kholer RA, Montemarano A. Dermatomyositis. Am Fam Physician 2001: 64: 1565-1572.

37 Alba MA, Flores-Suárez LF, Henderson AG, et al. Interstitial lung disease in ANCA vasculitis. Autoimmun Rev 2017; 16: 722-729.

38 Hoa S, Hudson M, Troyanov Y, et al. Single-specificity anti-Ku antibodies in an international cohort of 2140 systemic sclerosis subjects: clinical associations. Medicine 2016; 95: e4713

39 Ghirardello A, Borella E, Beggio M, et al. Myositis autoantibodies and clinical phenotypes. Auto Immun Highlights 2014; 5: 69-75.

40 Yamada H. ANCA: associated lung fibrosis. Semin Respir Crit Care Med 2011; 32: 322-327.

41 Nielen MM, van Schaardenburg D, Reesink HW, et al. Specific autoantibodies precede the symptoms of rheumatoid arthritis: a study of serial measurements in blood donors. Arthritis Rheum 2004; 50: 380-386.

42 Sambataro G, Sambataro D. Letter to editor. "Interstitial pneumonia with autoimmune features: clinical, radiologic and histological characteristics and outcome in a series of 57 patients". Respir Med 2017; 127: 65-66.

43 Ahmad K, Cottin V. The challenge of criteria for interstitial pneumonia with autoimmune features. Respir Med 2017; 127: 67.

44 Cutolo M, Sulli A, Smith V. How to perform and interpret capillaroscopy. Best Pract Res Clin Rheumatol 2013; 27: $237-248$

45 Sambataro D, Sambataro G, Zaccara E, et al. Nailfold videocapillaroscopy micro-haemorrhage and giant capillary counting as an accurate approach for a steady state definition of disease activity in systemic sclerosis. Arthritis Res Ther 2014; 16: 462 .

46 Andracco R, Irace R, Zaccara E, et al. The cumulative number of micro-haemorrhages and micro-thromboses in nailfold videocapillaroscopy is a good indicator of disease activity in systemic sclerosis: a validation study of the NEMO score. Arthritis Res Ther 2017; 19: 133.

47 Koenig M, Joyal E, Fritzler MJ, et al. Autoantibodies and microvascular damage are independent predictive factors for the progression of Raynaud's phenomenon to systemic sclerosis: a twenty-year prospective study of 586 patients, with validation of proposed criteria for early systemic sclerosis. Arthritis Rheum 2008; 58: 3902-3912.

48 Papaioannou AI, Kostikas K, Manali ED, et al. Combined pulmonary fibrosis and emphysema: the many aspects of a cohabitation contract. Respir Med 2016; 117: 14-26.

49 Cantin L, Bankier AA, Eisenberg RL. Bronchiectasis. AJR Am J Roentgenol 2009; 193: W158-W171. 